\title{
Iteratively Selecting Feature Subsets for Mining from High-Dimensional Databases
}

\author{
Hiroshi Mamitsuka \\ Institute for Chemical Research, Kyoto University \\ Gokasho, Uji, 611-0011, Japan \\ mami@kuicr.kyoto-u.ac.jp
}

\begin{abstract}
We propose a new data mining method that is effective for mining from extremely high-dimensional databases. Our proposed method iteratively selects a subset of features from a database and builds a hypothesis with the subset. Our selection of a feature subset has two steps, i.e. selecting a subset of instances from the database, to which predictions by multiple hypotheses previously obtained are most unreliable, and then selecting a subset of features, the distribution of whose values in the selected instances varies the most from that in all instances of the database. We empirically evaluate the effectiveness of the proposed method by comparing its performance with those of two other methods, including Xing et al.'s one of the latest feature subset selection methods. The evaluation was performed on a real-world data set with approximately 140,000 features. Our results show that the performance of the proposed method exceeds those of the other methods, both in terms of the final predictive accuracy and the precision attained at a recall given by Xing et al.'s method. We have also examined the effect of noise in the data and found that the advantage of the proposed method becomes more pronounced for larger noise levels.
\end{abstract}

\section{Introduction}

As the fields to which machine learning or data mining techniques are applied increase, the types of data sets dealt with have also been increasing. In particular, the growth of the size of the data sets in real world applications has been extremely pronounced. In this paper, among the large-scale data sets, we focus on mining from high-dimensional data sets, i.e. data sets with a large number (say 1,000,000) features (attributes), to which a single usual induction algorithm cannot be applied on a normal hardware. Our goal is to efficiently find prediction rules by mining from such a very high-dimensional data set. This type of data set, for example, appears in the process of drug design (or drug discovery). That is, each record in the data set corresponds to a chemical compound and has both an enormous number of features characterizing it and its label of drugability, toxicity etc..

So far, the only way to learn from this type of extremely high-dimensional data sets is to reduce the number of features of the data set by selecting a feature 
subset. There are two main techniques for feature subset selection, i.e. the filter and wrapper methods $[3,5,6]$. In both methods, there are two different approaches in terms of incremental or decremental selection of features. More concretely, the incremental approach starts with a set of no features and adds features one by one to the set, and the decremental approach starts with a set having all features and reduces the number of the features in the set. The incremental approach has to depend on the initial set of features, and thus, the decrementally selected features can be generally said to be more reliable than the incrementally selected ones. Actually, the recently proposed feature subset selection methods, e.g. $[4,8,12]$, belong to the decremental approach. Furthermore, an inductive algorithm used in the decremental wrapper approach has to be fed with the whole of a given high-dimensional data set at the initial process. Therefore, the decremental filter approach can be considered the most practical for the largesize data set considered here.

The method we propose here for a high-dimensional data set does not select a feature subset once, but iteratively selects a feature subset. Our method is closely related to an approach called 'sequential multi-subset learning with a model-guided instance selection', which is named in a comprehensive survey of [9] on the methods for up-scaling inductive algorithms. A method categorized in the approach repeats the following two steps: selecting a small subset of a large database, using previously obtained multiple predictive hypotheses, and training a component induction algorithm with the subset to obtain a new hypothesis. Our new method, named Qifs (Query-learning-based iterative feature-subset selection), follows the repetition of the two steps, but selects a subset of features from a given data set, instead of the subset of instances selected in the approach. Selecting a feature subset in Qifs consists of two steps. First, Qifs selects a subset of instances based on the idea of a method of query learning called 'Query by Committee' [11]. Using the idea, it predicts a label of each instance of the data set with existing hypotheses and selects the instances to which the predictions are distributed (or split) most evenly. Then, for each feature of the data set, Qifs focuses on the two distributions of feature values, i.e. the feature value distribution of the original all instances and that of the instances selected by the idea of query learning. Qifs selects the features in each of which the feature value distribution of the selected instances varies the most from that of the original all instances. Note that Qifs has the scalability for the number of features, as a method categorized in the above approach does for the size of data instances.

The Query by Committee algorithm selects the instances with maximum uncertainty of predicting label values so that the information gain in instance selection is maximized. In our scenario, the selected features are expected to be more strongly needed than others in terms of the information gain, since the distributions of their values in the selected instances differ the most from those in the original all instances, among all given features.

The purpose of this paper is to empirically evaluate the performance of Qifs using a data set used in KDD Cup 2001 which consists of approximately 140,000 binary features. In our experiments, we compared our new method with two 
other methods, including the latest feature subset selection method, proposed by [12]. We used two different component algorithms, C4.5 [10] and a support vector machine (SVM) [2] to test the performance for each of the three methods. Our evaluation was done by five-fold cross validation in all of our experiments.

We first used the original data set for the five-fold cross validation, and found that the performance of Qifs exceeds those of the other two methods in terms of final prediction accuracy. In order to better understand the conditions under which Qifs performs well, we varied the noise level of the original data set. That is, the noise was generated by randomly flipping the binary feature values and the noise level was controlled by varying the ratio of the number of flipped features to the number of all features. We evaluated the performance of the three methods, varying the noise level of the data set so that it was either ten or twenty percent. It was found that for larger noise levels, the significance level by which Qifs out-performed the other two methods in terms of final prediction accuracy became larger. Furthermore, we measured precision and recall (both of which are frequently used in information retrieval literature) of the three methods for the noisy data sets. In terms of the precision and recall, the difference between the precision value of Qifs and those of the other two methods at a same recall value also became larger, for larger noise levels.

All of these experiments show that for an extremely high-dimensional data set, our proposed method is more robust against noise than other currently-used methods for high-dimensional data sets and that our method will be a powerful tool for application to real-world very high-dimensional data sets.

\section{The Methods}

\subsection{Proposed Method}

Here, we propose a new method for mining from a very high-dimensional database. The method iteratively selects a feature subset and trains an arbitrary component algorithm as a subroutine with the subset. The method works roughly as follows. (See the pseudocode shown in Fig. 1.)

At the initialization, the method processes the following two different steps. For each feature, it calculates the distribution (number) of feature values in a given database (line 1 of Initialization). To obtain the first hypothesis, it uses an arbitrary algorithm of feature subset selection to obtain a feature subset and applies an arbitrary component learning algorithm to it. (line 2 of Initialization). At each iteration, it first calculates the 'margin' of each instance, that is, the difference between the number of votes by the past hypotheses for the most 'popular' label, and that for the second most popular label (line 1 of Step 1). It selects $N$ (say a half of all) instances having the least values of margin (line 2 of Step 1). Then, for each feature, it calculates the distribution of feature values in the selected instances and examines the 'difference' between the distribution of the selected instances and the previously calculated distribution of the whole given database (line 1 of Step 2). It selects a small (say 500) subset 
Input: Number of iterations: $T$

Component learning algorithm: $A$

Component feature subset selection algorithm: $B$

Set of features in a given data set: $F$

Set of instances in a given data set: $S$

Number of examples selected at each iteration: $N$

Training examples with a feature subset at the $i$-th iteration: $E_{i}$

Number of features in $E_{i}: Q$

Initialization: 1. For all $z \in F$, calculate the distribution $n_{z}$ of the feature values.

2. Select $Q$ features $\left\langle z_{1}^{+}, \cdots, z_{Q}^{+}\right\rangle$by running $B$ on the database

and obtain training instances $E_{1}=\left\langle z_{1}^{+}, \cdots, z_{Q}^{+}, y\right\rangle$ from database.

3. Run $A$ on $E_{1}$ and obtain hypothesis $h_{1}$.

For $i=1, \ldots, T$

Step1: 1 . For all $x \in S$, calculate 'margin' $m(x)$ using past hypotheses $h_{1}, \cdots, h_{i}$ $m(x)=\max _{y}\left|\left\{t \leq i: h_{t}(x)=y\right\}\right|-\max _{y \neq y_{\max }(x)}\left|\left\{t \leq i: h_{t}(x)=y\right\}\right|$

where $y_{\max }(x)=\arg \max _{y}\left|\left\{t \leq i: h_{t}(x)=y\right\}\right|$

2. Select $N$ instances having the smallest $m(x)$.

Step 2: $\quad 1$. For all $z \in F$, calculate distribution $n_{z}^{*}$ of the feature values in the selected instances, and calculate 'difference' $d_{z}$ between the two distributions, $n_{z}$ and $n_{z}^{*}$.

2. Select $Q$ features $\left\langle z_{1}^{+}, \cdots, z_{Q}^{+}\right\rangle$having the largest $d_{z}$ and let $E_{i+1}=\left\langle z_{1}^{+}, \cdots, z_{Q}^{+}, y\right\rangle$.

3. Run $A$ on $E_{i+1}$ and obtain hypothesis $h_{i+1}$.

End For

Output: Output final hypothesis given by: $h_{\text {fin }}(x)=\arg \max _{y \in Y}\left|\left\{t \leq T: h_{t}(x)=y\right\}\right|$

Fig. 1. Algorithm: Query-learning-based iterative feature-subset selection (Qifs)

$(Q)$ of features, whose calculated differences are the largest (line 2 of Step 2) and applies a component inductive algorithm to it to obtain a new hypothesis (line 3 of Step 2). The final hypothesis is defined by the majority vote over all the hypotheses obtained in the above process ${ }^{1}$.

The method first selects the instances that cannot be reliably predicted, and then selects features whose values in the selected instances differ the most from those of all the given instances. Since the method uses the technique of query learning in selecting the instances, we call our method 'Query-learning-based iterative feature-subset selection', Qifs for short.

The first step of Qifs, i.e. selecting instances, is almost the same as our previously proposed method, called QbagS [7]. QbagS is a method for mining from databases with a large number of instances and is also categorized in the approach named 'sequential multi-subset learning with a model-guided instance selection'. QbagS first randomly chooses a relatively large number of instances as selectable candidates from the database and then out of the candidates, selects instances to which predictions of previously obtained multiple hypotheses distributed (or split) most evenly, to build a new hypothesis. In [7], we have already shown that for very large and noisy datasets, QbagS out-performed Iv-

\footnotetext{
$\overline{1}$ Note that the total number of selected features equals to $T \times Q$.
} 
otes, one of the latest error-driven approaches, namely the methods which use label information and select examples on which the current hypotheses make a mistake.

In the second step of Qifs, a simple possible candidate for the difference between the distribution of the selected instances and that of all instances is the square distance, if a feature $z$ is a discrete attribute:

$$
d_{z}=\sum_{i}\left|\frac{n_{z}(i)}{\sum_{i} n_{z}(i)}-\frac{n_{z}^{+}(i)}{\sum_{i} n_{z}^{+}(i)}\right|^{2},
$$

where $d_{z}$ is the difference given to a feature $z, n_{z}(i)$ is the number of all instances in which the value of feature $z$ is $i$, and $n_{z}^{+}(i)$ is the number of the selected instances in which the value of feature $z$ is $i$. We used the difference given by Eq. (1), in our experiments.

Note that if a feature $z$ is a continuous attribute, we need to modify it to a discrete one to apply a distance (including the square distance described above) to it. There are actually a number of methods to discretize a continuous attribute, e.g. unconditional mixture modeling performed in [12].

\subsection{Xing et al.'s Feature Subset Selection Method}

Here, we briefly review Xing et al.'s feature selection method [12], to which we compare the performance of our method ${ }^{2}$. We can say that the method is one of the most recent feature subset selection methods, which belong to the decremental filter approach. The method consists of two steps, i.e. information gain ranking and Markov blanket filtering. The method first calculates the information gain for all given features and selects those which have a high information gain. Then, the selected features are reduced one by one using the method of Markov blanket filtering proposed by [4]. We can interpret the two steps roughly as follows: The information gain ranking of the method selects a set of features, each of which is strongly relevant to the label of a given database. Then, out of the obtained set, the Markov blanket filtering one by one removes the features for which a similar feature is contained in the set. Our implementation follows [12] exactly.

\section{Empirical Evaluation}

We empirically evaluate the performance of our method with those of two other methods, i.e. random iterative feature-subset sampling (hereafter called Rand for short) and Xing et al.'s feature subset selection method (hereafter called Fss for short). Random iterative feature-subset sampling is a strategy which, as done in Qifs, repeats the two steps of sampling a feature subset from a given database and applying a component learning algorithm to it, but it samples a

\footnotetext{
2 We also use Xing et al's method as our component feature-subset selection method, i.e. $B$ in Fig. 1
} 
Table 1. Data summary

Data set \# classes \# (binary) features \# training samples \# test samples

\begin{tabular}{lllll}
\hline Thrombin & 2 & 139,351 & 1560 & 390 \\
\hline
\end{tabular}

feature randomly when a feature subset is sampled. Thus, random feature-subset sampling does not use the previously obtained hypotheses and simply repeats random sampling and hypothesis building.

In our experiments, we used C4.5 [10] and a support vector machine (SVM) ${ }^{3}$ as a component learning algorithm. We used them with no particular options and equal conditions for all three methods, i.e. Qifs, Rand and Fss.

We need to evaluate our method with a data set which has a large number (say 100,000 or more) of features and to which we cannot apply a single usual inductive algorithm on a normal hardware. It is, however, difficult to find such a publicly available large-scale data set; the only one we found is a data set used in KDD Cup 20014. The KDD Cup data set is a real-world data set used for discovering drugs, i.e. small organic molecules which bind to a protein. The data set is a table in which each record, corresponding to a chemical molecule, has a number of binary (0 or 1$)$ features characterizing it and a binary class value of binding to a protein called 'thrombin'. A class value of 'A' for active (binding) and 'I' for inactive (non-binding) is given to each chemical compound. The sizes of the originally given training and test data sets are 1909 and 636, respectively. Out of them, we obtain a total of 1950 records (compounds) by mixing them, while removing the records in which all features are zero. Of the 1950, 190 are active compounds. That is, the percentage of the compounds binding to thrombin is $9.74 \%$. We call the data set 'Thrombin data set'.

In evaluating the Thrombin data set, we compare the 'final accuracy' obtained by each of the methods. By final accuracy, we mean an accuracy level large enough that the predictive performance appears to be saturating ${ }^{5}$. We also used the measures of precision and recall, standard performance measures in the field of information retrieval. Note that 'recall' is defined as the probability of correct prediction given that the actual label is ' $\mathrm{A}$ ', and 'precision' is defined as the probability of correct prediction given that the predicted label is ' $\mathrm{A}$ '.

In all of the experiments, the evaluation was done by five-fold cross validation. That is, we split the data set into five blocks of roughly equal size, and in each trial four out of these five blocks were used as training data, and the last block was reserved for test data. The results (learning curves, final accuracy, precision and recall) were then averaged over the five runs.

\footnotetext{
${ }^{3}$ To be precise, we used $\mathrm{SVM}^{\text {light }}[2]$.

${ }^{4}$ http://www.cs.wisc.edu/ dpage/kddcup2001/

${ }^{5}$ To be exact, we compared the predictive accuracy obtained at a certain point with that obtained 1,000 seconds prior to that point. If the difference between them is less than $0.05 \%$, we considered that the predictive accuracy at that point is saturated.
} 
Table 2. Summary of parameter settings in our experiments

\begin{tabular}{lrr}
\hline \multicolumn{3}{c}{ \# selected features \# selected samples } \\
Methods & per iteration $(Q)$ & per iteration $(N)$ \\
\hline Qifs & 500 & 780 \\
Rand & 500 & - \\
\hline
\end{tabular}
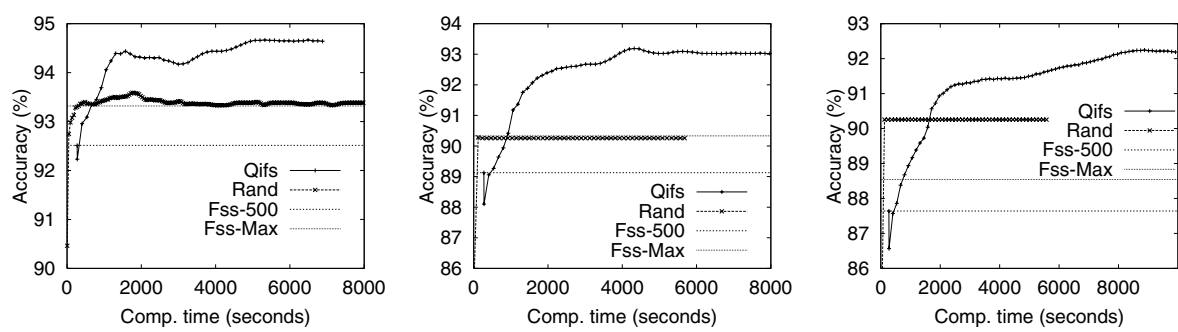

Fig. 2. Learning curves of Qifs and Rand using C4.5 as a component algorithm, with the prediction accuracies of Fss shown for reference, when the noise level is (a) zero, (b) ten and (c) twenty percent

The properties of the Thrombin data set used in our five-fold cross validation are shown in Table 1.

The parameters of Qifs and Rand that were used in our experiments are shown in Table 2. In all of our experiments, we run Fss as follows: We first reduce the number of features to 1,000 by choosing the top features as ranked by information gain, and then we reduce the number one by one to 100 by Markov blanket filtering. In the latter process, we check the prediction accuracy (on separate test data) of a feature set whenever it is obtained, and thus, we obtain a total of 901 subsets and prediction accuracies.

\subsection{Cross-Validation on Thrombin Data Set}

We show the results of the cross-validation on the Thrombin data set in the form of learning curves in Figures 2 and $3^{6}$.

Figures 2 (a) and 3 (a) show the learning curves using C4.5 and an SVM as a component learning algorithm, respectively. Note that, in these curves, the average prediction accuracy (on separate test data) is plotted against the total computation time, including disk access time. In both Figures 2 and 3, we also

\footnotetext{
6 The number of iterations of Qifs till the average prediction accuracy is saturated varies widely among the cases of Figures 2 and 3 . It ranges from approximately fifty to a couple of hundreds.
} 

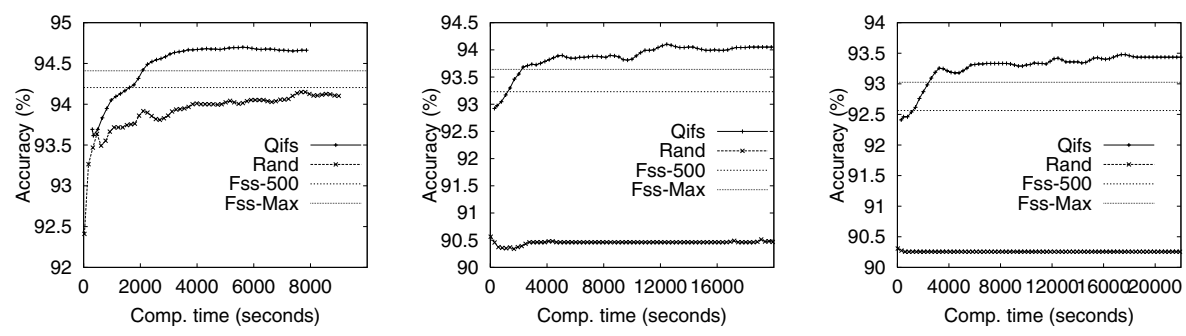

Fig. 3. Learning curves of Qifs and Rand using an SVM as a component algorithm, with the prediction accuracies of Fss shown for reference, when the noise level is (a) zero, (b) ten and (c) twenty percent

add two types of prediction accuracies for Fss for reference ${ }^{7}$. One, shown as Fss500 , is the accuracy obtained when the number of selected features reaches 500 , which is the same number as that of the selected features at each iteration in both Qifs and Rand, and the other, shown as Fss-Max, is the highest accuracy attained while reducing the number of features from 1,000 to 100 . Note that in a practical situation, we cannot obtain the accuracy given by the Fss-Max for unknown test data, and thus, the performance of our method should be compared with that of not Fss-Max but Fss-500.

In terms of the 'final prediction accuracy' results, Qifs out-performed both Rand and Fss. These results are summarized in Table 3, as the case of the noise level of zero percent. The accuracies reached by the three methods for the data set and the ' $t$ ' values of the mean difference significance (pairwise) test for the respective cases are given in the table. The $t$ values are calculated using the following formula :

$$
t=\frac{|\operatorname{ave}(D)|}{\sqrt{\frac{\operatorname{var}(D)}{n}}},
$$

where we let $D$ denote the difference between the accuracies of two methods for each data set in our cross-validation, ave $(X)$ the average of $X, \operatorname{var}(X)$ the variance of $X$, and $n$ the number of data sets (five in our case). For the case that $n=5$, if $t$ is greater than 4.604 then it is more than 99 per cent statistically significant that one achieves higher accuracy than the other.

As is shown in Table 3, for the Thrombin data set, the $t$ values range from 1.15 to 4.78 . We can statistically see that the performance of Qifs is slightly (insignificantly) better than those of Rand and Fss.

In order to check the performance of our method in more realistic conditions, we add a kind of noise to the Thrombin data set, varying the noise level. More concretely, we randomly reversed binary feature values of the data set, while

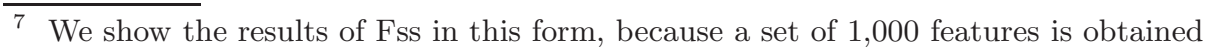
all at once in the first step (information gain ranking) of Fss and thus learning curves cannot be obtained from the process of feature subset selection.
} 
Table 3. Average final accuracies of Qifs and Rand, average accuracies of Fss-500 and Fss-Max, and the $t$ values calculated between Qifs and Rand and between Qifs and Fss-500

\begin{tabular}{c|c|c|c|c|c|c|c}
\hline Noise & Component & \multicolumn{4}{|c|}{ Final accuracy (\%) } & $t$ & $t$ \\
\cline { 3 - 6 } level(\%) & algorithm & Qifs & Rand & Fss-500 & Fss-Max & (vs. Rand) & (vs. Fss-500) \\
\hline 0 & C4.5 & $\mathbf{9 4 . 6 7}$ & 93.38 & 92.51 & 93.33 & 4.56 & $\mathbf{4 . 7 8}$ \\
& SVM & $\mathbf{9 4 . 6 6}$ & 94.12 & 94.41 & 94.21 & 2.55 & 1.15 \\
\hline 10 & C4.5 & $\mathbf{9 3 . 0 3}$ & 90.26 & 89.13 & 90.31 & $\mathbf{8 . 4 7}$ & $\mathbf{1 1 . 2 8}$ \\
& SVM & $\mathbf{9 4 . 0 5}$ & 90.46 & 93.64 & 93.23 & $\mathbf{6 . 0 5}$ & 2.34 \\
\hline 20 & C4.5 & $\mathbf{9 2 . 2 1}$ & 90.26 & 87.64 & 88.51 & $\mathbf{7 . 7 7}$ & $\mathbf{7 . 4 2}$ \\
& SVM & $\mathbf{9 3 . 4 4}$ & 90.26 & 92.56 & 93.03 & $\mathbf{1 1 . 1 0}$ & $\mathbf{6 . 1 1}$ \\
\hline
\end{tabular}

keeping the percentage of the number of the reversed features at a certain level, i.e. ten or twenty percent. Figures 2 (b) and (c) show the learning curves of the ten and twenty percent noise level, respectively, using $\mathrm{C} 4.5$ as a component algorithm. Figures 3 (b) and (c) also show the learning curves of the two noise levels, using an SVM as a component algorithm. Here, too, in terms of the final prediction accuracy results, Qifs performed better than both Rand and Fss. The final prediction accuracies and the $t$-values of the mean difference significance test for the cases of the noise levels of ten and twenty percent are also summarized in Table 3.

When the noise level is ten or twenty percent, Qifs did significantly better than Rand or Fss in seven out of the eight cases, in terms of the $t$ values shown in Table 3. We can see, in these results, that for higher noise level, the significance of the difference in the predictive performance between Qifs and the other two methods becomes more pronounced. This result can be visualized by the graph shown in Figure 6 (a). The figure shows how the $t$ values of the mean difference significance test vary as the noise level is changed from zero to twenty percent. We also found from the results that the difference between the performance of Qifs and those of Rand and Fss obtained by using C4.5 as a component learning algorithm, is larger than that obtained by using an SVM.

The precision-recall curves for Qifs, Rand and Fss are shown in Figures 4, using C4.5 as a component learning algorithm. Note that in Fss, prediction is done by a single induction algorithm and only a single pair of recall and precision values is obtained. For Qifs and Rand, the precision-recall curves shown in Figure 4 are those attained after approximately (a) 6,000 (b) 2,000 and (c) 5,000 seconds of computation time. The curves in Figure 5 are those attained after approximately (a) 7,000 (b) 18,000 and (c) 20,000 seconds of computation time using an SVM as a component algorithm.

As shown in the figure, for larger noise levels, the gap between the precision value of Qifs at a certain recall value and those of the other two methods at the same recall value is larger. In particular, as shown in Figure 4 (c), when the noise level reaches twenty percent, the precision of Qifs is approximately 40 percent 

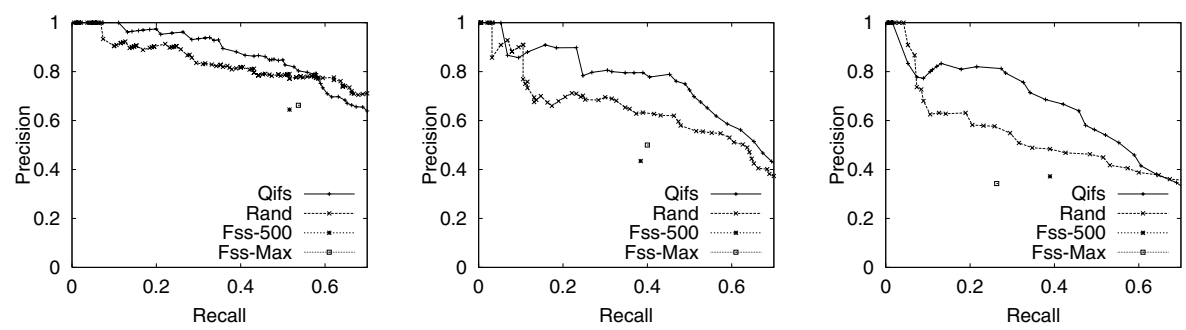

Fig. 4. Precision-recall curves of Qifs, Rand and Fss using C4.5 as a component algorithm, when the noise level is (a) zero (b) ten and (c) twenty percent
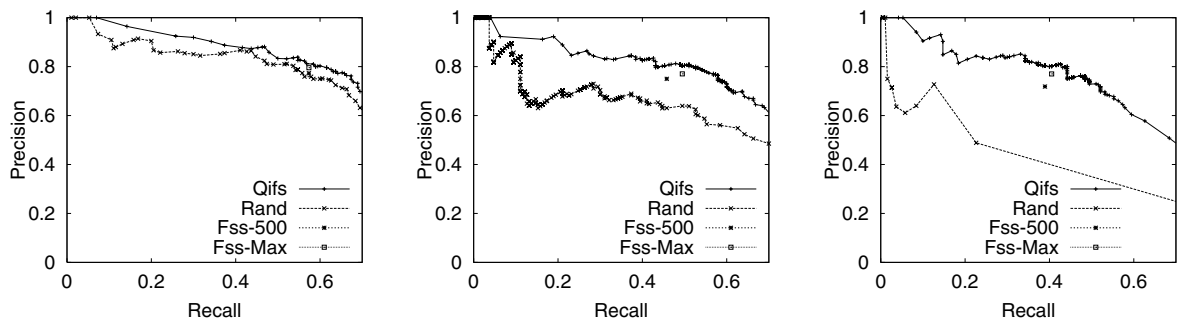

Fig. 5. Precision-recall curves of Qifs, Rand and Fss using an SVM as a component algorithm, when the noise level is (a) zero (b) ten and (c) twenty percent

better than that of Fss-500 at an equal recall value given by Fss-500. One more item of note is that the performance of Rand is better than that of Fss, in the case of using C4.5 as a component learning algorithm. This shows that there is a case in which multiple hypotheses built by sets of randomly selected features achieve a better predictive performance than the single hypothesis built by a set of features carefully selected from all given features.

The results of precision-recall curves for all three methods when using either C4.5 or an SVM as a component learning algorithm, are summarized in Table 4. The table shows, for each noise level and component algorithm, the precision values of the three methods at a recall value given by Fss-500. This result can be visualized by the graph shown in Figure 6 (b). The figure shows how the ratio of precision values of Rand or Fss-500 to those of Qifs varies as the noise level is changed from zero to twenty percent.

\section{Concluding Remarks}

We have proposed a new method for data mining that targets the mining from very high-dimensional, noisy data sets. Though the number of features of the data set used here is 140,000 , we have shown that the performance of our pro- 
Table 4. Precision values of Qifs, Rand and Fss-500, corresponding to recall values given by Fss-500

\begin{tabular}{c|c|c|c|c|c}
\hline algorithm & level(\%) & Qifs & Rand & Fss-500 & (Fss-500) \\
\hline C4.5 & 0 & $\mathbf{0 . 8 2 8 ( 1 . 0 )}$ & $0.790(0.954)$ & $0.645(0.779)$ & 0.516 \\
& 10 & $\mathbf{0 . 7 9 6 ( 1 . 0 )}$ & $0.632(0.794)$ & $0.434(0.545)$ & 0.384 \\
& 20 & $\mathbf{0 . 6 8 6 ( 1 . 0 )}$ & $0.484(0.706)$ & $0.372(0.542)$ & 0.389 \\
\hline SVM & 0 & $\mathbf{0 . 8 1 1 ( 1 . 0 )}$ & $0.757(0.933)$ & $0.773(0.953)$ & 0.574 \\
& 10 & $\mathbf{0 . 8 0 4 ( 1 . 0 )}$ & $0.630(0.784)$ & $0.75(0.933)$ & 0.458 \\
& 20 & $\mathbf{0 . 8 0 4 ( 1 . 0 )}$ & $0.41(0.510)$ & $0.718(0.893)$ & 0.389 \\
\hline
\end{tabular}
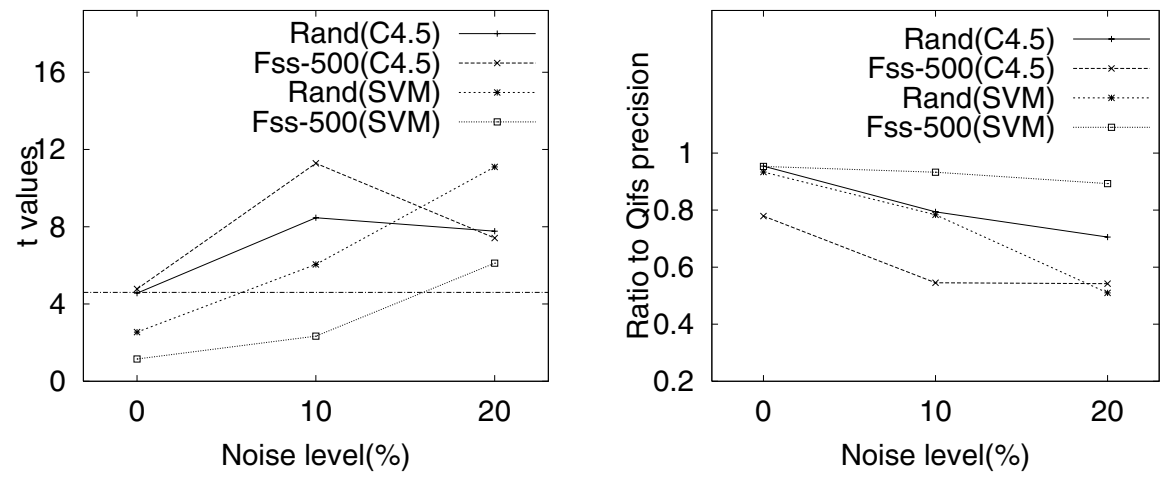

Fig. 6. (a) $t$ values obtained when varying the noise level and (b) ratio of precision of Rand/Fss to that of Qifs obtained when varying the noise level

posed method is clearly better than that of one of the latest feature subset selection method. The advantage of our method would become more pronounced for more high-dimensional and noisy data sets. The key property of our method which contributes to this advantage is its iterative feature-subset sampling strategy, based on the idea of query learning.

We may compare Qifs with another method which iteratively selects feature subsets using Fss. More concretely, it repeats as follows: it first randomly picks a subset of a given data set, then runs Fss for the subset and obtains a hypothesis with the final feature subset and a learning algorithm. Final prediction is done by the majority votes of the obtained hypotheses. This comparison may be a possible interesting future work.

For mining from a large database which has a large number of both features and instances, we can modify our method to a selective sampling method, in which we use only the instances obtained by the first step of our current method to build a new hypothesis. That is, the new method iteratively selects a subset of both instances and features from the large-scale database. It would also 
be interesting to investigate under what conditions (noise level and number of features and instances) it works better than other methods, if such a type of database is available.

\section{Acknowledgements}

The author would like to thank Naoki Abe of IBM for discussions related to the topics of this paper and anonymous reviewers for helpful comments.

\section{References}

1. Breiman, L.: Pasting Small Votes for Classification in Large Databases and On-line. Machine Learning 36 (1999) 85-103

2. Joachims, T. Making Large-scale SVM Learning Practical. In: Scholkopf, B., Burges, C., Smola, A. (eds.): Advances in Kernel Methods - Support Vector Learning, B. MIT Press, Cambridge (1999) 363, 366

3. Kohavi, R., John, G. H.: Wrappers for Feature Subset Selection. Artificial Intelligence 97 (1997) 273-324 362

4. Koller, D., Sahami, M.: Toward Optimal Feature Selection. In: Saitta, L. (eds.): Proceedings of the Thirteenth International Conference on Machine Learning. Morgan Kaufmann, Bari, Italy (1996) 284-292 362, 365

5. Kononenko, I., Hong, S. J.: Attribute Selection for Modelling. Future Generation Computer Systems 13 (1997) 181-195 362

6. Liu, H., Motoda, H.: Feature Selection for Knowledge Discovery Data Mining. Kluwer Academic Publishers, Boston (1998) 362

7. Mamitsuka, H., Abe, N.: Efficient Mining from Large Databases by Query Learning. In: Langley, P. (eds.): Proceedings of the Seventeenth International Conference on Machine Learning. Morgan Kaufmann, Stanford Univ., CA (2000) 575-582 364

8. Ng, A.: On Feature Selection: Learning with Exponentially Many Irrelevant Features as Training Examples. In: Shavlik, J. (eds.): Proceedings of the Fifteenth Intenational Conference on Machine Learning. Morgan Kaufmann, Madison, WI (1998) 404-412 362

9. Provost, F., Kolluri, V.: A Survey of Methods for Scaling up Inductive Algorithms. Knowledge Discovery and Data Mining 3 (1999) 131-169 362

10. Quinlan, J. R.: C4.5: Programs for Machine Learning. Morgan Kaufmann, San Francisco (1993) 363, 366

11. Seung, H. S., Opper, M., Sompolinsky, H.: Query by Committee. In: Haussler, D. (eds.): Proceedings of the Fifth Intenational Conference on Computational Learning Theory. Morgan Kaufmann, NY (1992) 287-294 362

12. Xing, E. P., Jordan, M. I., Karp, R. M.: Feature Selection for High-dimensional Genomic Microarray Data In: Brodley, C. E., Danyluk, A. P. (eds.): Proceedings of the Eighteenth Intenational Conference on Machine Learning. Morgan Kaufmann, Madison, WI (2001) 601-608 